\title{
Instruções de higiene na escola e na sociedade como ação de saúde e prevenção de doenças: uma revisão bibliográfica
}

\author{
Hygiene instructions at school and in society as a health action and disease prevention: a \\ bibliographic review
}
Instrucciones de higiene em la escuela y em la sociedad como acción de salud y prevención de enfermedades: una revisión bibliográfica

\begin{abstract}
Lázaro Saluci Ramos ${ }^{1 *}$, Hilda Angélica Lima Fontana Gomes², Thaís Cardoso Guimarães de Aguiar $^{2}$, Rozária Maria dos Santos Soares ${ }^{2}$, Mateus Xavier Corrêa ${ }^{2}$, Luciana Tonon Fontana Morgan², Jamylle Chaves Mota², Cristiane Aparecida Chaves Mota², Kaline de Almeida Queiroz², Alessandra Luzia da Gama Cotta².
\end{abstract}

\section{RESUMO}

Objetivo: Objetiva-se nesta revisão reunir toda pesquisa realizada na expectativa de introduzir ou aprimorar, conhecimentos de higiene aos alunos, como alternativa a uma nova política de saúde na escola. Revisão Bibliográfica: As escolas formam um grande núcleo de conhecimentos específicos. Todas as áreas possuem desdobramentos, e dentro das disciplinas próximas da área da saúde, um tema pouco desenvolvido e abordado é a higiene. Nas escolas concentra-se maior esforço nos conhecimentos científicos e é notório um desprezo de como poderá ser empregado este conhecimento em situações do cotidiano. As disciplinas da área da saúde são a chave para introduzir a cultura da higiene profunda e segura, e mostrar como que ampliar e difundir os métodos é importante para prevenir doenças e garantir saúde, não somente no ambiente escolar, mas introduzir a matéria na sociedade através da instrução dos discentes. Considerações finais: A análise busca incessantemente demonstrar a importância da aplicação do conhecimento em higiene na sociedade e como a difusão nas escolas pode ampliar a distribuição dessa pauta na sociedade, através de uma ação de saúde em sala de aula.

Palavras-chave: Higiene, Saúde corporal, Saúde do aluno.

\begin{abstract}
Objective: The objective of this review is to gather all research carried out with the expectation of introducing or improving hygiene knowledge to students, as an alternative to a new health policy at school. Bibliographic Review: Schools form a large core of specific knowledge. All areas have developments, and within the disciplines close to the health area, a poorly developed and addressed theme is hygiene. In schools, a greater effort is concentrated on scientific knowledge and there is a disregard for how this knowledge can be used in everyday situations. Health disciplines are the key to introducing the culture of deep and safe hygiene, and show how expanding and spreading methods is important to prevent disease and ensure health, not only in the school environment, but to introduce the subject into society through instruction of students. Final considerations: The analysis constantly seeks to demonstrate the importance of applying knowledge on hygiene in society and how the diffusion in schools can expand the distribution of this agenda in society, through a health action in the classroom.
\end{abstract}

Keywords: Hygiene, Body health, Student health.

${ }^{1}$ Faculdade Multivix (MULTIVIX), Cachoeiro de Itapemirim - ES. *E-mail: lazaro321123@gmail.com

${ }^{2}$ Faculdade Vale do Cricaré (FVC), São Mateus - MG. 


\section{RESUMEN}

Objetivo: El objetivo de esta revisión es reunir toda la investigación realizada con la expectativa de introducir o mejorar el conocimiento de higiene a los estudiantes, como una alternativa a una nueva política de salud en la escuela. Revisión Bibliográfica: Las escuelas forman un gran núcleo de conocimiento específico. Todas las áreas tienen desarrollos, y dentro de las disciplinas cercanas al área de la salud, un tema poco desarrollado y abordado es la higiene. En las escuelas, un mayor esfuerzo se concentra en el conocimiento científico y es notorio el desprecio de cómo se puede usar este conocimiento en situaciones cotidianas. Las disciplinas de salud son la clave para introducir la cultura de la higiene profunda y segura, y muestran cómo es importante expandir y difundir los métodos para prevenir enfermedades y garantizar la salud, no solo en el entorno escolar, sino para introducir el tema en la sociedad a través de instrucción de estudiantes. Consideraciones finales: El análisis busca constantemente demostrar la importancia de aplicar el conocimiento sobre higiene en la sociedad y cómo la difusión en las escuelas puede expandir la distribución de esta agenda en la sociedad, a través de una acción de salud en el aula.

Palabras clave: Higiene, Salud corporal, Salud estudiantil.

\section{INTRODUÇÃO}

O conceito de higiene está intimamente ligado a saúde. O seu conceito traz um significado bem específico em táticas de promover a saúde através da limpeza. Foi na Idade Média que as pessoas começaram a suspeitar que uma substância "sólida" poderia espalhar doenças de uma pessoa para outra. O médico italiano Francastorius de Verona descreveu a epidemia em seu livro De Contagione e mencionou a infecção. Segundo as informações dos marinheiros que testemunharam a propagação da doença durante a expedição colombiana, ele declarou que a doença era causada pela disseminação de microrganismos de pessoa para pessoa. Em 1546, Francastorius sustentou a ideia de que doenças eram transmitidas através de corpúsculos, ele chamou de sementes da doença (seminário primário), e essas sementes eram por contato direto ou por roupas e objetos. Passe de um corpo para outro (FONTANA RT, 2006).

Para controlar doenças e melhorar a vida social, a atenção das pessoas às cidades e à saúde urbana e a conversão delas em um conjunto de regras e leis são o resultado de uma longa jornada histórica. A higienização como prática médica e científica pode ser rastreada até o início do século XIX. Entende-se como "a arte de preservar a vida" e indica a direção de seu uso, seja o conceito daqueles que confirmam a responsabilidade pessoal no processo de doença e morte ou aqueles que são determinados pela sociedade. Como medida para reduzir o desperdício de recursos humanos causado por doenças, a higienização também é conhecida como um "guia científico". Como objeto de estudo, destacou-se na Alemanha em meados do Século XIX, estabelecendo as bases para o progresso e o renascimento social (LAROCCA LM e MARQUES VRB, 2010).

As crianças são uma parte importante da saúde coletiva, tanto porque os adultos precisam "cuidar", quanto porque os agravos e hábitos que ocorrem ou começam nesta fase podem ter um impacto em suas vidas. Além disso, durante a infância, existem várias mudanças, principalmente as físicas e psicológicas, características do crescimento e desenvolvimento das crianças. Além dos fatores socioeconômicos, a cultura também afeta o ambiente familiar e comunitário e pode ser definida como um grupo de grupos específicos de padrões, crenças e valores de comportamento de aprendizagem. A higiene corporal inclui cuidados com o corpo, cabelos, dentes e unhas, incluindo a limpeza das mãos. O Ministério da Saúde recomenda tomar banho e escovar os dentes após uma refeição todos os dias. 6 Além de trazer conforto e melhorar a aparência pessoal, o banho também pode remover a sujeira e reduzir o número de micróbios na pele (REMOR CB, et al., 2009).

As doenças parasitárias na infância têm uma relação profunda com os determinantes sociais e ambientais, portanto, em áreas com condições instáveis de moradia, alto saneamento básico e alto suprimento de água, a prevalência de parasitas é alta. Reconhecendo que existem muitas razões para esse problema, a educação em saúde se tornou uma estratégia importante para o desenvolvimento de bons hábitos de higiene. 
Os profissionais de saúde devem sempre considerar a educação em saúde como um conteúdo constante e existe em suas diretrizes nos ambientes escolar e doméstico e tomar medidas preventivas no manuseio de alimentos, no consumo de água e no uso de boas práticas de higiene. Quando um indivíduo é instruído, ele/ela tem o direito de decidir questões relacionadas à saúde, para que ele/ela possa tomar ações críticas e refletir sua atitude (NASCIMENTO JS, 2015).

A escola é geralmente uma extensão da família, principalmente quando oferecer educação infantil. Eles repetem seus hábitos e aprendizados em casa, por isso é importante desenvolver certos hábitos. Portanto, é essencial incentivar a higiene escolar, não apenas comentar ou ensinar. Os hábitos de higiene escolar devem ser fortalecidos, desde ensinar as crianças a lavar as mãos e escovar os dentes até explicar a importância de lavar as frutas antes de comer. Em termos de higiene escolar, cuidar de água e comida no refeitório da escola também é uma questão importante. Utilizar as disciplinas específicas de ciências para introduzir práticas de higiene e reforçar os cuidados com a limpeza, surte como processo indispensável a manutenção da saúde do aluno (CARVALHO PHA, et al., 2016).

O objetivo desta revisão apresentar em consonância com a bibliografia existente a importância de introduzir nas escolas práticas de higiene corporal à fim de expandir o alcance dessas práticas, e utilizar os alunos como meio de transmissão desse conhecimento para as futuras gerações. É de igual interesse, explorar as disciplinas voltadas ao campo da saúde para difundir no cotidiano escolar conhecimentos e novas práticas, aumentando os cuidados e prevenindo enfermidades.

\section{REVISÃO BIBLIOGRÁFICA}

O ambiente escolar, além de ser um espaço fértil da disseminação de conhecimentos, é o local onde melhor pode-se encontrar espaço para inserir na sociedade novas práticas e novos hábitos. O conhecimento tem por finalidade ampliar linhas de pesquisa que fortalecem a longevidade e a facilitação das atividades humanas na busca por prazer e qualidade. Todo conhecimento disseminado nas escolas tem também por objetivo desenvolver práticas na sociedade, entretanto pouco se vê o ambiente escolar incentivando a cultura dos bons hábitos, tanto pelos métodos ultrapassados que mecanizaram a educação, quanto pela falta de recursos, tempo e preparação ideal fornecida aos profissionais docentes (CARVALHO FR e WATANABE G, 2019).

No momento quando as palavras dos higienistas começaram a girar em torno da educação binomial e elucidação da saúde, o Instituto de Hygiene também constituiu um espaço importante na elucidação de estratégias voltadas à comunicação de mensagens de saúde no universo escolar. O desempenho no treinamento profissional dos professores do ensino fundamental inclui tanto o treinamento do pessoal de saúde pública quanto a produção de materiais impressos destinados ao uso de outras audiências para os alunos do ensino fundamental e seus professores.

A obediência pessoal aos requisitos de saúde é configurada como uma fórmula que se apresenta ao espírito das pessoas, observando e investigando os problemas causados pelo rápido crescimento urbano e pelas irregularidades da população. Parar as torrentes mórbidas é indispensável a enorme ameaça que paira na sociedade e um desafio para os cientistas higienistas (ROCHA HHP, 2003).

O impacto das condições básicas de saneamento na saúde da população é bem conhecido. Para as crianças, essas doenças estão diretamente relacionadas à mortalidade e morbidade infantil assim como os problemas de saúde estão relacionados à renda da população. O Brasil é um dos que mais registram no mundo a incidência de doenças causadas por parasitas e vermes, e as maiores vítimas são as crianças. A higiene pessoal é um tópico envolvido e estudado na educação não formal, incluindo os cuidados diários que os indivíduos devem realizar com seus próprios corpos. Os hábitos de higiene não se limitam a tomar banho ou escovar os dentes após cada refeição, mas também prestar atenção à dieta, beber água filtrada e outros comportamentos que ajudam a manter a saúde física e mental (CRUZ JDS, 2018). 
A higiene na escola está inteiramente ligada, principalmente, a questões alimentares e nutricionais. A importância de agir, com o uso de formas lúdicas que ajudem as crianças a aprender a importância de uma dieta saudável e limpa, a fim de buscar uma melhora no estado nutricional, tornou-se óbvia. Nesse sentido, ao realizar atividades de saúde por meio de atividades criativas, sejam elas de prevenção ou de promoção da saúde, pode-se promover maior grau de interação e fortalecer relacionamentos, para que as ações atendam às necessidades e expectativas do discente. Para comprovar tal situação, as condições de saúde das escolas foram avaliadas, assinalou-se que, em termos de higiene, apenas $68 \%$ apresentavam condições normais, $51 \%$ apresentavam tinea pedis, $70 \%$ apresentavam danos à dentição, $39 \%$ atrasavam a vacinação e 38\% desnutrição grau I, 26\% estão no grau II (GUETERRES EC, et al., 2017).

A imunização é a medida mais eficaz para prevenir doenças gripais. Todos os anos, as pessoas que são consideradas mais suscetíveis aos vírus recebem vacinas gratuitas. No entanto, o número de subtipos de vírus da gripe que constituem imunobiologia é limitado a cada ano. Portanto, além de monitorar os dados de circulação de vírus para reduzir os casos de doenças, também é necessário tomar medidas preventivas e de controle (AMORIM CSV, et al., 2019).

Dentre essas medidas, apresentam-se algumas como higiene das mãos antes das refeições, espirros ou tosse, use tecidos descartáveis para higiene nasal, evite o contato com a mucosa ocular, boca e cavidade nasal e evite compartilhar itens pessoais com outras pessoas, como pratos, copos, louças e manutenção de um ambiente bem ventilado são essenciais para prevenir infecções.

Também é recomendável suprimir a saída de casa durante o período de alta taxa de transmissão e limitar o contato próximo com pessoas que apresentam sintomas ou sinais de gripe. Também se recomenda afastamentos temporários de atividades laborais e escolares (AMORIM CSV, et al., 2019).

\section{A expansão das práticas de higiene nas escolas}

Ao praticar hábitos de higiene pessoal todos os dias, não apenas evita-se infecções causadas principalmente por fungos e bactérias, mas também é possível melhorar as impressões pessoais ou profissionais de pessoas relacionadas a nós. De acordo com esse princípio, hábitos de higiene pessoal devem ser desenvolvidos desde o início, a fim de conscientizar todos os jovens e adultos brasileiros. Portanto, é necessário refletir sobre os métodos atuais adotados pelos pais, educadores e profissionais de saúde para aumentar a conscientização sobre a necessidade de compreender esse conteúdo, tornando esse ensino mais persuasivo (SILVA LFS e ALVES NC, 2014).

O problema da higiene escolar apareceu nas últimas décadas do século XIX. Em 15 de março de 1884, em uma revista didática de Portugal (ano 1, ano 2), e nela estava: A aplicação da higiene só é estudada nos tempos modernos. Às escolas, principalmente às escolas primárias, desde o método usado para a escola do prédio da escola, o professor terá que cuidar de todas as situações que possam ajudar a confiar nos cuidados com o crescimento da criança. Um decreto estipulou claramente que o Estado é responsável pelo desenvolvimento físico e manutenção da saúde das crianças, o que as obriga a frequentar a escola durante parte de sua infância (RODRIGUES MM, 2014).

A Declaração de Bogotá propõe, entre outras coisas, condições adequadas para a criação de conhecimento, com a participação da comunidade educacional, isso pode facilitar o uso de estilos de vida e comportamentos saudáveis para proteger o meio ambiente. A importância disso foi claramente demonstrada e explicada em várias conferências internacionais sobre esse tema.

No Brasil, as pessoas também começaram a perceber a importância da promoção da saúde na escola. Até 1996, de acordo com a resolução da Lei de Diretrizes e Bases da Educação Nacional (LDBEN) 5692, o tema saúde era discutido no âmbito do currículo escolar, mas não era incluído no currículo (GONÇALVES FD, et al., 2008).

De acordo com o Conselho Federal de Educação (CFE) n- 2.264 / 74, o objetivo desta declaração é orientar crianças e adolescentes a desenvolver hábitos saudáveis relacionados à higiene pessoal, alimentação, esportes, trabalho e lazer, para que possam ser utilizados imediatamente para manter a saúde de indivíduos e outras pessoas.

REAS / EJCH | Vol.12(10) | e4558 | DOI: https://doi.org/10.25248/reas.e4558.2020 Página 4 de 7 
As práticas encorajadas nas escolas devem fortalecer os princípios da dignidade da pessoa humana, aumentando o acesso aos direitos básicos e indispensáveis para a promoção da saúde do ser humano, e facilitando $o$ acesso da comunidade nas decisões dos rumos a serem tomados no ambiente escolar. A forma de difundir o conhecimento proposto é trazendo para perto as famílias dos alunos (GONÇALVES FD, et al., 2008).

Uma das discussões centrais acerca do desenvolvimento das políticas de higiene nas escolas, além do incentivo a aplicação no cotidiano, é o espaço escasso dentro das casas de educação no Brasil. Já que a maior parte das escolas brasileiras ainda não funcionam em tempo integral, o que dificulta o aprimoramento da educação básica e a ampliação da grade, de forma que possa contemplar disciplinas não formais e promover cultura e lazer na escola. As disciplinas orientadas a trabalhar com o tema da higiene são, no ensino fundamental as disciplinas de Ciências e Educação Física, e no ensino médio as disciplinas de Biologia, Química, Física e Educação Física. Entretanto, essas disciplinas não possuem uma grade apurada e coesa com os objetivos sociais ao redor da temática (LEITE LP, et al., 2011).

Dentro da educação ambiental, que tem sido fortalecida devido ao crescimento do interesse na preservação do meio ambiente, muito se toca nas questões referentes ao combate à poluição, coleta seletiva e a utilização em menor número de produtos nocivos a natureza. Questões como o descarte correto do lixo, cuidado com os alimentos e precauções ao frequentar ambientes possivelmente contaminados, podem se expandir e dar espaço a questões da higiene pessoal e como ela é importante para a preservação da saúde humana (PINHÃO F e MARTINS I, 2012).

Também na educação infantil, se faz necessário incluir tratamentos com a higiene e outros cuidados com o corpo humano. Há a expectativa que na fase de $0-5$ anos, a criança tenha seus primeiros contatos e desenvolva noções básicas alimentares e higiênicas, à fim de que o constante progresso no tratamento das áreas esteja completo antes mesmo do fim da adolescência. É de importância ímpar garantir que novos hábitos sejam encorajados no ambiente escolar, e que a cultura despreocupada com a higiene corporal seja fortalecida. Somente dessa forma será possível utilizar o ambiente escolar para disseminar um conhecimento indispensável para a sociedade e garantir controle sobre a transmissão de doenças virais (CRESPO MR, 2013).

\section{A higiene na prevenção de doenças}

As mudanças sociais, econômicas e demográficas que ocorreram nos últimos 50 anos foram os fatores que determinam as mudanças significativas nos padrões globais de morbimortalidade. No contexto dessas mudanças, expandir a cobertura das instalações de saneamento, melhorar as condições da moradia e adotar novas tecnologias de assistência à saúde, especialmente vacinas e antibióticos, é decisivo para o rápido declínio no número de doenças infecciosas. Essa nova situação despertou otimismo nas décadas de 60 e 70 do Século XX, de que, com a maioria dos países alcançando amplo desenvolvimento econômico e acesso a melhores condições de vida, esse grupo de doenças perderá sua importância na saúde pública (WALDMAN EA e SATO APS, 2016).

Ainda nos anos 60 e 70, as doenças infecciosas foram particularmente importantes na agenda de saúde pública. Entre eles, as chamadas doenças endêmicas rurais. A discussão versou sobre a saúde da população rural no Brasil, apontando a prevalência de desnutrição e fome crônica condições de moradia e saneamento e sua completa falta de instalações de saneamento e higiene (PESSOA SB, 1963). A rápida industrialização do país e a urbanização que se seguiu, o desenvolvimento econômico, a expansão da educação em todos os níveis e a instituição de um sistema unificado de saúde mudaram. Especialmente nas últimas três décadas a estrutura de saúde do Brasil foi projetada para eliminar a malária e condições propícias a doenças endêmicas rurais, inserindo-as na agenda prioritária de saúde pública rural (WALDMAN EA e SATO APS, 2016).

As doenças transmitidas por alimentos tornaram-se a causa de investigação e pesquisa a fim de que a etiologia e os fatores relacionados possam ser identificados e analisados. A compreensão dos fatores envolvidos no processo é importante para o estabelecimento de mecanismos de prevenção e controle. 
O Brasil não possui legislação para estabelecer padrões microbiológicos para esse tipo de análise, mas a presença de bactérias indica ausência de higiene das mãos e defeitos sanitários, o que representa risco a saúde de toda sociedade. Entende-se, portanto, que os hábitos de limpeza, tanto corporal quanto alimentares, são a chave para exercer controle e prevenir adoecimento por contaminação (MEDEIROS MGGA, et al., 2017).

As percepções acerca da prevenção de doenças através da higiene foi objeto de pesquisa comparativa colonização de protógenos respiratórios. Um grupo de pacientes internados em uma Unidade de Terapia Intensiva (UTI) foi comparado a um grupo de pacientes que recebeu tratamento clínico de rotina na clínica odontológica. Entre os pacientes de UTI, a higiene bucal é pior que os pacientes clínicos. Placa e lou mucosa oral de $65 \%$ dos pacientes em UTI foram colonizadas por patógenos respiratórios, em comparação com apenas $16 \%$ dos pacientes clínicos. Concluiu-se, portanto, que a placa dentária pode ser um importante repositório de patógenos respiratórios, e a melhoria da higiene bucal em pacientes hospitalizados pode reduzir a placa dentária e as colônias orofaríngeas (KAHN S, et al., 2008).

A saúde é um estado de total felicidade, ou seja, aspectos físicos, psicológicos e sociais são muito bons. Ao contrário do que muitas pessoas pensam, a saúde é indispensavelmente destinada aos indivíduos e comunidades e está fundamentalmente relacionada à qualidade de vida das comunidades e famílias. Cuidados básicos com o corpo, como tomar banho, cortar cabelos, manter as unhas aparadas, escovar os dentes, lavar as mãos e também apresentar-se bem higienizado ajuda na prevenção de doenças. Para esse fim, devemos buscar uma posição-chave nas linhas finas de saúde, higiene e doença (SCHOT AG, et al., 2016).

O cuidado com a higiene é uma oportunidade para permitir que os enfermeiros diagnostiquem a necessidade mais mencionada de cuidados privilegiados. Durante todo o processo de assistência médica as pessoas podem fazer parte de duas maneiras diferentes: tornar-se parte do todo, participar ativamente ou simplesmente receber atendimento. O paciente é uma parte indispensável e tem um sentimento de pertencer a ele. Os cuidados com o banho nas casas de saúde, se referem ao tempo que o enfermeiro passa com as pessoas, onde ele tem a oportunidade de se comunicar pessoalmente com o paciente, para que ele possa coletar informações, para que o cuidado com o banho sanitário se torne uma oportunidade, pois além dos cuidados que fornecem proteção contra infecções, há também os cuidados que permitem ao paciente se sentir integrado (FONSECA EF, et al., 2015).

\section{CONSIDERAÇÕES FINAIS}

Compreende-se, portanto, que a prevenção de doenças está intimamente ligada a práticas de higiene pessoal e hospitalar. As casas de saúde pública e seus profissionais exercem papel fundamental na saúde do ser humano quando cuidam da limpeza do seu ambiente, e que a melhor maneira de difundir essas práticas e conhecimentos é introduzindo na educação básica brasileira instruções de higiene pessoal, garantindo que os atores das futuras gerações integrem o grupo de difusores das boas práticas. É dessa maneira que permitirse-á uma comunicação maior entre saúde e educação no intuito de solucionar problemas sociais.

\section{REFERÊNCIAS}

1. AMORIM CSV, et al. Higiene das mãos e prevenção da Influenza: Conhecimento de discentes da área da saúde. Texto \& Contexto - Enfermagem, 2019; 27(4): 1-9.

2. CARVALHO FR, WATANABE G. A construção do conhecimento científico escolar: Hipóteses de transição identificadas a partir das ideias doas (as) alunos(as). Educação em Revista, 2019; 35(1): 1-16.

3. CARVALHO PHA, et al. Avaliação de uma proposta educativa em saúde bucal aplicada ao ensino básico. Odontologia Clínico-Científica (Online), 2016; 15(1): 1-10.

4. CRESPO MR. Saúde na escola sob a perspectiva das professoras de educação infantil. Trabalho de Conclusão de Curso (Educação Física). Universidade Estadual de Campinas, Campinas - São Paulo, 2013; p. 50.

5. CRUZ JDS. Higiene pessoal como uma proposta inovadora para melhorar a qualidade de vida de crianças e adolescentes que fazem parte do Centro de Apoio Lar Peniel na cidade de Simão Dias-SE. 9o Simpósio Internacional de Educação e Comunicação, 2018: 1-16. 
6. FONTANA RT. As infecções hospitalares e a evolução histórica das infecções. Revista Brasileira de Enfermagem, 2006; 59-5: 703-706.

7. FONSECA EF, at al. Cuidados de higiene - banho: significados e perspectivas dos enfermeiros. Revista de Enfermagem Referência, 2015; 4(5): 37-45.

8. GUETERRES EC, et al. Educação em saúde no contexto escolar: estudo de revisão integrativa. Enfermería Global, 2017; 46(2): 477-488.

9. KAHN S, et al. Avaliação da existência de controle de infecção oral nos pacientes internados em hospitais do estado do Rio de Janeiro. Ciência \& Saúde Coletiva, 2008; 13(6): 1825-1831.

10. LAROCCA LM, MARQUES VRB. Higienizar, cuidar e civilizar: o discurso médico para a escola paranaense (19201937). Interface (comunicação, saúde e educação), 2010; 14(34): 647-660.

11. LEITE LP, et al. A adequação curricular como facilitadora da educação inclusiva. Psicologia da Educação, 2011; 1(32): 89-111.

12. MEDEIROS MGGA, et al. Percepção sobre a higiene dos manipuladores de alimentos e perfil microbiológico em restaurante universitário. Ciência \& Saúde Coletiva, 2017; (22)2: 383-392.

13. NASCIMENTO JS. Adesão de hábitos de higiene em crianças no ambiente escolar de uma comunidade da zona rural do Município de Junqueiro - Alagoas. Trabalho de conclusão do curso Especialização em Estratégia e Saúde da Família. Universidade Federal de Minas Gerais, Belo Horizonte - Minas Gerais, 2015; 29 p.

14. PESSOA SB. Endemias parasitárias da zona rural brasileira. São Paulo (SP): Fundo Editorial Procienx; 1963.

15. PINHÃO F e MARTINS I. Diferentes abordagens sobre o tema saúde e ambiente: desafios para o ensino de ciências. Ciência \& Educação (Bauru), 2012; 18(4): 819-836.

16. REMOR CB, et al. Percepções e conhecimentos das mães em relação às práticas de higiene de seus filhos. Pesquisa, Research - Investigación, 2009; 13(4): 786-92.

17. ROCHA HHP. Educação escolar e higienização da infância. Cad. Cedes, 2003; 23(59): 39-56.

18. RODRIGUES MM. A higienização da escola primária portuguesa no amanhecer do século 20. História da Educação, 2014; 18(42): 75-92.

19. SCHOT AG, et al. Higiene como princípio básico de uma boa saúde. XXIV Seminário Internacional de Educação SIEDUCA, 2016; 1(1): 1-5.

20. SILVA LFS, ALVES NC. Higiene Pessoal: a importância de estudar o corpo humano. Biodiversidade, 2014; 13(2): 7589.

21. WALDMAN EA, SATO APS. Trajetória das doenças infecciosas no Brasil nos últimos 50 anos: um contínuo desafio. Revista de Saúde Pública, 2016: 50-68. 